\title{
Programa Saúde da Família no Brasil: uma agenda incompleta?
}

\author{
Family $\mathrm{H}$ ealth Program in Brazil: an incomplete agenda?
}

M aria Fátima de Sousa ${ }^{1}$

Edgar M erchán Hamann ${ }^{1}$

${ }^{1} \mathrm{Núcleo} \mathrm{deEstudos} \mathrm{de}$

SaúdePública, Centro de

EstudosAvançados

Multidisciplinares, UnB.

SCLN 406, BI. A, Sala 223.

70847-510 Brasília DF.

mariafatimasousa@uol.com.br

Abstract The present article is a longitudinal analysis of the Brazilian experience with the Family $\mathrm{H}$ ealth Program (PSF) throughout its 12 years of implementation. The article starts with the fundamental purpose of such strategy which is the reorganization of Basic Health Care, contextualizing in the light of this unique and peculiar experience to the Brazilian Unified Health System (SUS). It highlights the progresses and challenges of the PSF, indicating urgent needs regarding technological incorporation, formation of labor force, establishment of new mechanisms as well as agreements on basic care funds and the direction of new strategies and technologies in the process of monitoring and evaluation. To conclude, the article shows the elements to foster a debate about such questions, which must be permanent and lucid in the perspective of building the Brazilian U nified Health System and the right to health.

Key words Primary care, Basic Care, Family H ealth, Brazilian Unified $\mathrm{H}$ ealth System
Resumo Esteartigo trata deuma análiselongitudinal da experiência brasileira com o Programa Saúde da Família (PSF), ao longo dos doze anos da sua implantação e implementação. Toma como ponto de partida o propósito fundamental desta estratégia, que éa reorganização da atenção básica de saúde, contextualizando à luz da experiência que é única e peculiar ao Sistema Ú nico deSaúde(SUS). Evidencia os avanços e desafios do PSF, apontando nesse sentido as necessidades mais urgentes em termos de incorporação tecnológica, formação da força de trabalho, estabelecimento de novos mecanismos e pactos em torno do financiamento da atenção básica e encaminhamento de novas estratégias e tecnologias nos processos de monitoramento e avaliação. Finaliza apontando elementos para subsidiar um debate sobre as questões em pauta, o qual deve ser permanentee lúcido na perspectiva da construção do SUS e do direito à saúde.

Palavras-chave Atenção primária, Atenção básica, Saúde da Família, Sistema Ú nico de Saúde 
Introdução

O objetivo deste artigo é analisar o Programa de Saúdeda Família (PSF), uma experiência brasileira, que ao longo dos dez anos da sua implantação e implementação, vem constituindo-se como uma estratégia para a reorganização da atenção básica à saúde.

Criado em março de 1994 como um programa, uma década depois de sua implantação e funcionamento, o PSF evoluiu dessa condição e vem assumindo 0 status de uma política nacional que conta com 26.100 equipes de saúde da família, às quais se vinculam 216.055 agentes comunitários de saúde que prestam assistência a 83,8 milhões de pessoas em 5.274 municípios brasileiros ${ }^{1}$.

Como um componente constituinte do Sistema Ú nico de Saúde (SUS), o PSF configura as dimensões das práticas de saúde características da sociedade brasileira em termos territoriais, populacionais e das ações desenvolvidas nesses territórios. Ademais, constitui uma das resultantes das mobilizações que caracterizaram esta mesma sociedade na construção do direito à saúde ao longo de mais de quatro décadas. A saúde como um direito, portanto, não é um objeto de estudo recente no Brasil, haja vista a presença histórica de atores sociais como o M ovimento Sanitário Brasileiro e o campo da Saúde Coletiva, que viabilizaram a constituição de sujeitos ao longo dessas décadas. Estes atores configuraram no campo da saúde uma prática social com plena expressividade das suas dimensões técnicas, políticas e ideológicas, ao ritmo do processo de democratização da sociedade brasileira. As experiências desenvolvidas em municípios como M ontes Claros (M G), Londrina (PR), Campinas (SP) e outros ${ }^{2-5}$ revelam a possibilidade de questionar, na prática, a concepção da saúde restrita à dimensão médico-assistencial. Seguramente as experiências destesmunicípiosalicerçaram as bases conceituais, técnicas e ideológicas do desenho em escala nacional do Programa de Agentes Comunitários de Saúde (PACS) e do Programa de Saúde da Família (PSF) que vêm sendo implantadas no Brasil desde 1991 e1994, respectivamente.

Em outras palavras, estas estratégias nascem no acirramento da crise estrutural do setor saúde no país, vivenciada pela população brasileira, que por muitas décadas sente o distanciamento entre seus direitos garantidos em leis ${ }^{6-8}$ e a capacidade efetiva do Estado de ofertar as ações e serviços públicos de saúde, o mais perto possível dos territórios sóciosanitários dos indivíduos, famílias e comunidades.

Tal realidade impulsionou em diferentes conjunturas e governos, ao longo dos últimos doze anos, a conjugação de esforços para reunir as capacidades nacionais ( gestores públicos do Sistema Único de Saúde, professores, pesquisadores, corporações, outros) em torno de uma agenda pactuada e integrada para responder às demandas da população como condição essencial ao enfrentamento da crise paradigmática do modelo clássico de atenção à saúde no Brasil, com foco na atenção básica. E que a atenção básica seja o primeiro contato de um modelo de atenção longitudinalmente focalizado na família, com orientações comunitárias, coordenado eintegrado sistemicamente ${ }^{9}$, para que neste nível a atenção à saúde seja de al ta qualidade, efetiva e equitativa.

Trabalhar com modelo de atenção à saúde voltado à comunidadenão étarefa simples, pois exige um olhar voltado para os grupos sociais específicos, supõe um rompimento dos muros dos serviços de saúde e, sobretudo, um alto grau de complexidade do conhecimento. No dizer de $M$ endes ${ }^{10}$, as tecnologias empregadas na atenção básica são de menor densidade e maior complexidade, uma vez queseutilizam, por um lado, recursos de baixo custo no que se refere a equipamentos diagnósticos e apoios terapêuticos; por outro lado, incorporam instrumentos tecnológicos advindos das Ciências Sociais (Antropologia, Sociologia e H istória) e Humanas (Economia, Geografia, etc.) na compreensão do processo saúde- doença e na intervenção coletiva eindividual.

\section{A tenção primária e atenção básica à saúde}

O termo atenção básica à saúde é pouco encontrado na literatura mundial. 0 conceito usual para este campo de práticas é atenção primária à saúde, como decorrência do pacto mundial realizado em 1978, na cidade de Alma-Ata (República de Cazaquistão), na antiga União Soviética (URSS), onde diversos países participaram da Conferência Internacional deCuidados Primários deSaúdeeafirmaram compromissos com a meta de "saúde para todos no ano 2000", constituindo assim a Declaração de Alma-Ata.

Tal evento apontava também para a atenção primária como sendo composta de cuidados essenciais de saúde baseados em métodos e tecnologias práticas, cientificamente bem fundamentados esocialmenteaceitáveis, colocados ao alcanceuniversal de indivíduos e famílias da comunidade, mediante sua plena participação.

Reforçava, para tanto, a saúde como sendo não a simples ausência de doença, mas como o "estado de completo bem-estar físico, mental esocial", tra- 
zendo para o âmbito formal einternacional aquilo que $M$ arc Lalonde, ministro da Saúde do Canadá, já vinha apontando como os componentes básicos para a abordagem da saúde dos povos: "a biologia humana, o meio ambiente, o estilo de vida ea organização da atenção à saúde" ${ }^{\prime 1}$. Em síntese, pode-se afirmar que o pensamento da atenção primária no mundo apresenta-se com vários sentidos desde o primeiro nível de atenção, passando pela idéia de um tipo de serviços, uma filosofia de atendimento, até uma estratégia de organização dos sistemas de saúde.

No caso brasileiro, o termo atenção básica significa o primeiro nível deatenção à saúdee sustenta-se no princípio da integral idade, compreendido como a articulação de ações de promoção da saúde e prevenção, tratamento e reabilitação dedoenças e agravos. É, portanto, uma formulação típica do SUS, que deixa claro os seus princípios e suas diretrizes organizativas e é incorporado pela estratégia do PSF, a qual, a partir de sua criação, materializa uma forma de pensar e agir na construção de um novo modelo de atenção à saúde dos indivíduos, famílias e comunidades.

Nesta direção, após quatro anos de implantação do PSF, o M inistério da Saúde, por intermédio da Secretaria de Assistência à Saúde/Coordenação da Atenção Básica, impulsionou o debate, com a responsabilidade técnica-política de pactuar entre as três instâncias de governos (gestores do SUS) suas competências nestenível deatenção. Esta iniciativa resulta na publicação da Portaria no $3.925^{12}$. Assim, no ano seguinte, o manual foi lançado como resultado de um pacto tripartite, firmando com isto o conceito da atenção básica, definido como um conjunto de ações, de caráter individual ou coletivo, situadas no primeiro nível de atenção dos sistemas de saúde, voltado para a promoção da saúde, prevenção de agravos, tratamento e reabilitação. A ampliação desse conceito se torna necessária para avançar na direção de um sistema de saúde centrado na qualidade de vida das pessoas e de seu meio ambiente.

Passados oito anos e considerando a necessidade de revisar e adequar as normas nacionais ao atual momento do desenvolvimento da atenção básica no Brasil, o Ministério da Saúde aprova a Política Nacional deAtenção Básica ${ }^{13}$, estabelecendo a revisão de diretrizes e normas para a organização da atenção básica para o Programa Saúde da Família (PSF) eo Programa Agente Comunitários de Saúde (PACS), em que reafirma o conceito da atenção básica e explicita quea mesma deve ser desenvolvida por meio do exercício de práticas gerenciais e sanitárias democráticas e participativas, sob forma de trabalho em equipe, dirigidas a populações de territórios bem delimitados. E, ainda, que se devem utilizar tecnologias de el evada complexidade e baixa densidade, de forma a resolver os problemas de saúde de maior frequência e relevância em seu território.

Isto posto, pode-se afirmar, seja no cenário internacional ou nacional, que o conceito de atenção primária e/ou atenção básica sedirige paraum ponto comum, em que seus princípios e diretrizes gerais, de caráter planetário, têm sido nestas últimas décadas o balizador da formulação de estratégias para a construção de um novo modelo de atenção à saúde, cujas bases se sustentam em práticas cuidadosas, resolutivas e qualificadas. Portanto, suas diferenças dar-se-ão na medida da especificidade sócio-cultural e política de cada nação e serão pautadas pelas relações que se estabel ecem entre Estado e sociedade em cada contexto.

\section{PSF : a estratégia política}

para reorganizar a atenção básica

O Programa de Saúde da Família, desde sua origem, foi concebido como uma estratégia para a reorganização e fortalecimento da atenção básica como o primeiro nível de atenção à saúdeno SUS, mediante a ampliação do acesso, a qual ificação e a reorientação das práticas de saúde.

O caráter substitutivo do PSF em relação à "atenção básica tradicional" orienta-se pelos seguintes princípios: 1) adscrição de clientela; 2) territorialização; 3) diagnóstico da situação de saúde da população e 4) planejamento baseado na realidade local. A adscrição da clientela refere-se ao novo vínculo que se estabelece de modo permanente entre os grupos sociais, as equipes e as unidades de saúde. A territorialização aponta para a relação precisa quese estabelecemediantea definição do território e da população, o que implica o mapeamento e a segmentação da população por território. 0 diagnóstico da situação de saúde da população permite a análise da situação de saúde do território, mediante cadastramento das famílias e dos indivíduos ea geração de dados. 0 planejamento baseado na realidadelocal viabiliza a programação de atividades orientada segundo critérios de risco à saúde, priorizando solução dos problemas em articulação permanente com os indivíduos, famílias e comunidades.

As características do processo de trabalho das equipes multiprofissionais compostas por, no mínimo, médico, enfermeiro, auxiliar deenfermagem ou técnico de enfermagem e agentes comunitários 
de saúde, responsáveis por cuidarem em média de 3.000 habitantes, com jornada de trabal ho dequarenta horas semanais, passam, necessariamente, pela interdisciplinaridade, vinculação, competência cultural, intersetorialidade e fortalecimento de uma gestão local que deve ser participativa/democrática. Passam, sobretudo, pela singularidade da presença dos agentes comunitários em saúde na equipe. A intenção é que a conversão do modelo faça a diferença na forma de pensar e de fazer no cotidiano, a saúde das famílias, tanto nos aspectos da promoção, prevenção, recuperação e reabilitação de doenças e agravos.

Segundo Campos et al. ${ }^{14}$, o PSF no Brasil, mesmo baseado nas idéias de atenção primária, pre venção de doenças e promoção da saúde aceitas internacionalmente, éoriginal einédito em sua concepção, não havendo registro de modelos seme Ihantes em outros países. Diferentemente da me dicina familiar vigente em vários países da Europa, no Canadá e na O ceania, o PSF pressupõe o trabaIho multiprofissional e em equipe como processo básico para a integralidade do cuidado na atenção primária à saúde.

Nesta direção, a Portaria $n^{\circ} 648$, de 28 de março de 2006, reforça os objetivos do PSF, quando explicita, mais uma vez, suas responsabilidades sanitárias no conjunto do sistema: (a) possibilitar o acesso universal e contínuo a serviços de saúde de qualidade e resolutivos, caracterizados como a porta de entrada preferencial do sistema de saúde, com território adscrito de forma a permitir o planejamento e a programação descentralizada, e em consonância com o princípio da equidade; (b) efetivar a integralidade em seus vários aspectos, a saber: integração de ações programáticas e demanda espontânea; articulação das ações de promoção à saúde, prevenção de agravos, vigilância à saúde, tratamento e reabilitação, trabalho de forma interdisciplinar e em equipe, e coordenação do cuidado na rede de serviços; (c) desenvolver relações de vínculo e responsabilização entre as equipes e a população adscrita, garantindo a continuidade das ações de saúde e a longitudinalidade do cuidado; (d) valorizar os profissionais de saúde por meio do estímulo e do acompanhamento constante de sua formação e capacitação; (e) realizar avaliação e acompanhamento sistemático dos resultados alcançados, como parte do processo de planejamento e programação; e (f) estimular a participação popular e o controle social.

Para Teixei ra ${ }^{15}$, com a reafirmação desses objetivos, o PSF deixa de ser um programa que operacional izava uma política de focalização da atenção básica em populações excluídas do consumo de serviços para ser considerado uma estratégia de mudança do modelo de atenção à saúde no SUS, na verdade, o instrumento de uma política de universalização da cobertura da atenção básica, e, portanto, um espaço de reorganização do processo de trabalho em saúde nesse nível. Com essa condição, o PSF entra no cenário nacional, após seis anos deconstituição do SU S, enesse contexto emergem as discussões em torno do modelo de atenção à saúde, ocupando um espaço de maior destaque, até então concedido para as questões relativas ao financiamento, à descentralização e à participação social.

Deve-se reconhecer que, por um lado, a conjuntura era favorável para discussão de novas formas de organização de serviço, tendo no PSF sua base estruturante; por outro, estávamos em plena década de noventa, marco das políticas neoliberais na América Latina eno Brasil. Essecenário fez com que alguns autores considerados importantes no movimento sanitário brasileiro julgassem ser o PSF um programa pontual, "pacote básico" de assistência à saúde, cujas bases se sustentavam em um projeto- piloto centrado no médico, direcionado à clientela específica e focalizado em regiões pobres.

$\mathrm{Na}$ questão da focalização ou universalização, compartilhamos da compreensão de outros autores quando vêem na universalização o princípio ético básico, consistindo em garantir a todos os cidadãos, independentemente de gênero, etnia, nível de renda, vinculação de trabalho ou nível de risco, os direitos sociaisfundamentais, em quantidadeequalidade compatíveis com o grau de desenvolvimento de uma determinada sociedade. A universalização não é contrária ao estabelecimento de critérios de seletividade ou focal ização, desdequea sel etividade ou a focalização estejam subordinadas a uma política geral de universalização. Assim, mesmo a seletividade pode ser utilizada como um instrumento de política social, mas não pode converter-se na política social, porque nesse caso estaria quebrando o princípio da universalização.

Viana e Dal Poz ${ }^{16}$ têm posições próximas e dizem: [...] experiências de focalização dentro do universalismo evidenciam que se pode ter práticas focalizadas dentro de uma política universal e não há necessariamente conflito entre focalização e universalização. Pode-se afirmar que há uma convergência entre os posicionamentos desses autores e nós, quando entendemos ser o PSF uma estratégia política que vem ao longo dos doze anos de implantação, e implementação, contribuindo para a diminuição das iniquidades, sobretudo para a ampliação do acesso aos serviços básicos de saúde e vinculação das equipes junto aos indivíduos, fa- 
mílias e comunidades, na complexa tarefa de cuidar da vida, saúde, doença, sofrimento e morte.

N esse sentido, cabereconhecer na trajetória do PSF alguns avanços, apontando para o enfrentamento dos desafios atuais. Portanto, merecem destaque, ainda que no limite desse artigo, com vista ao aprofundamento futuro, as seguintes questões: a expansão da cobertura do PSF para os grandes centros urbanos; a co-responsabilidade do financiamento da atenção básica/PSF; a política de capacitação, formação e educação permanente para o pessoal do Saúde da Família; todas entrelaçadas pela necessidade deum maior investimento na produção e inovação tecnológica aplicada aos processos de monitoramento e avaliação dos resultados dos processos organizativos das Unidades Básicas de Saúde que operaram na lógica dessa estratégia.

\section{A expansão da cobertura do PSF} para os grandes centros urbanos

O movimento de expansão do PSF demonstra uma progressiva adesão dos gestores municipais e estaduais, sobretudo no co-financiamento, por meio de criação de incentivos estaduais, o que não se via em 1994, ano de sua implantação. Naquela época, somente doze estados da federação aderiram a essa iniciativa (Alagoas, Ceará, Paraíba, Pernambuco, Sergipe, Pará, Goiás, M inas Gerais, Rio de Janeiro, São Paulo, Paraná e Santa Catarina), e doze municípios, considerados pioneiros: Bragança (PA), Campina Grande (PB), Neópolis (SE), Quixadá (CE), Goiânia e Planaltina de Goiás (GO), Juiz de Fora e Além Paraíba (MG), Niterói (RJ), Curitiba (PR), Joinvillee Criciúma (SC). A decisão políticae determinação técnica desses municípios fizeram com que, ao final de 1994, o PSF estivesse presenteem 55 cidades brasileiras com 328 equipes atuando.

0 marco de sua expansão ocorreu no ano de 1998, como resultado da mudança do financiamento com a implantação do Piso da Atenção Básica (PAB), em 1997. Posteriormente, foi reforçada pela renovação dos gestores locais de saúde em 2001 eo início das negociações em torno do Pacto da Atenção Básica. Ao final daquele ano, o PSF encontrava-se implantado em 1.134 municípios. No ano que antecede a implantação do PAB, a cobertura era de 5.599,350 milhões de pessoas, o que representava 3,5\% da população brasileira, distribuída em municípios de pequeno porte - entre 10 e 20 mil habitantes - e médio porte - abaixo de 50 mil habitantes, evidenciando uma baixa adesão dos municípios de grande porte.

Ao longo dos doze anos de implantação dessa estratégia, o número de equipes vem expandindose progressivamente e, segundo dados do Sistema de Informação da Atenção Básica (SIAB), atualmentesão 26.259, quecuidam diariamente, em diferentes espaços do território brasileiro, de 84,2 milhões de pessoas em 5.100 municípios, representando $45 \%$ do total da população. No país, conta-se com a presença de 217.117 agentes comunitários de saúde, em 5.274 municípios, os quais visitam, em média, uma vez ao mês, 108,9 milhões de pessoas, $58,5 \%$ da população brasileira, população esta excluída historicamente do acesso aos bens de serviços e ações do setor saúde.

Ainda que reconhecendo a expansão progressiva das equipes de saúde da família nos municípios de todas as regiões do país, verifica-se uma desigualdade na cobertura do PSF entre as regiões e nos chamados grandes centros urbanos (municípios acima de 100 mil habitantes, e capitais), onde mais de $50 \%$ da população brasileira residem. Portanto, nas capitais, salta aos olhos a baixa cobertura das equipes do PSF.

A Tabela 1 mostra a desigualdade já mencionada no texto, sobretudo nos municípios de $\mathrm{Ma-}$ naus, Salvador, Fortaleza, Belém, Curitiba, Rio de Janeiro, Porto Alegre, São Paulo e Braślia/Distrito Federal, cidades que concentram o maior número de pessoas, entre as demais capitais.

Essas desigualdades resultam das dificuldades já verificadas na implementação do PSF em municípios de pequeno emédio porte, que são potencialmente agravadas nas grandes cidades e metrópoles. Consideram-se nestas grandes cidades fatores contribuintes para a não implantação do PSF, tais como os altos níveis de exclusão no acesso aos serviços de saúde, os agravos de saúde característicos dos grandes centros, a oferta consolidada de uma rede assistencial desarticulada e mal distribuída, a predominância da modalidade tradicional de atendimento à demanda e de programas verticalizados sem estabelecimento de vínculos com a comunidade do seu território ${ }^{17}$.

Vários autores assinalam a obtenção de boas práticas por parte das equipes do PSF, sobretudo nos municípios de pequeno porte (Teixeira, Sousa, inter alia). Porém, muitas vezes estas práticas apresentam-se limitadas para a oferta de atenção integral face à ausência de uma rede regionalizada de referência e contra-referência de serviços assistenciais. Logo, não alteram substantivamente a lógica organizativa dos serviços e sistemas locais de saúde, predominando com isto o modelo clássico de assistência a doenças em suas demandas espontâneas, sustentadas no tripé do médico (consultas, apoio diagnóstico), equipamentos e medicamentos. 
Tabela 1. N úmero de equipes do PSF nas capitais e cobertura populacional, 2006.

\begin{tabular}{llrrrr}
\hline UF & Município & População & No Equipes do PSF & População Coberta & Cobertura \% \\
AC & Rio Branco & 309.731 & 40 & 138.000 & 44,6 \\
AL & M aceió & 309.731 & 40 & 138.000 & 44,6 \\
A & Manaus & 1.648 .218 & 130 & 448.500 & 27,2 \\
AP & Macapá & 356.228 & 31 & 106.950 & 30,0 \\
BA & Salvador & 2.673 .560 & 125 & 431.250 & 16,1 \\
CE & Fortaleza & 2.374 .944 & 202 & 696.900 & 29,3 \\
DF & Braślia & 2.334 .322 & 42 & 144.900 & 6,2 \\
ES & Vitória & 313.312 & 51 & 175.950 & 56,2 \\
GO & Goiânia & 1.201 .006 & 118 & 407.100 & 35,5 \\
M A & São Luís & 980.926 & 89 & 307.050 & 31,3 \\
M G & Belo Horizonte & 2.375 .329 & 504 & 1.738 .800 & 73,2 \\
M S & Campo Grande & 750.424 & 48 & 165.600 & 22,1 \\
M T & Cuiabá & 536.000 & 30 & 103.500 & 19,3 \\
PA & Belém & 1.406 .355 & 82 & 282.900 & 20,1 \\
PB & João Pessoa & 660.798 & 172 & 593.400 & 89,8 \\
PE & Recife & 1.501 .008 & 216 & 745.200 & 49,6 \\
PI & Teresina & 791.341 & 211 & 727.950 & 92,0 \\
PR & Curitiba & 1.757 .904 & 141 & 486.450 & 30,6 \\
RJ & Rio dejaneiro & 6.094 .183 & 115 & 396.750 & 6,5 \\
RN & Natal & 778.040 & 104 & 358.800 & 46,1 \\
RO & Porto Velho & 382.093 & 28 & 96.600 & 25,3 \\
RR & Boa Vista & 249.903 & 53 & 182.850 & 73,2 \\
RS & Porto Alegre & 1.428 .696 & 90 & 310.500 & 21,7 \\
SC & Florianópolis & 396.778 & 78 & 269.100 & 67,8 \\
SE & Aracaju & 498.619 & 128 & 441.600 & 88,6 \\
SP & São Paulo & 10.927 .985 & 818 & 2.822 .100 & 25,8 \\
TO & Palmas & 208.793 & 39 & 134.550 & 64,4 \\
Total & & 43.840 .181 & 3.757 & 12.961 .650 & 29,6 \\
\hline & & & & & \\
\end{tabular}

Fonte: Dados do Sistema de Informação da Atenção Básica (SI AB), agosto de 2006.

Para análise dessa situação, Teixeira ${ }^{18}$ nos instiga sobre a pertinência de expandir essa estratégia para os municípios acima de 100.000 habitantes, da mesma forma como vem sendo implementada nos demais. Nesses novos espaços-territórios, o PSF passa a concorrer fortemente com as práticas assistenciais médico-curativas, o que provoca tensão e resistência de atores envolvidos na manutenção do modelo hegemônico.

Adiciona-se a esse quadro a disputa políticopartidária entreestados emunicípios, quando suas gestões estão sob o comando de partidos políticos contrários. Nesses cenários, as resistências e oposição corporativa e ideológica se ampliam, também, quando as cidades contam com uma rede física instalada centralizada nos hospitais e ambulatórios de subespecialidades. Outros fatores que contribuem para 0 acirramento dessas resistências residem em recursos de apoio diagnóstico e terapêutico concentrados na região central, as agendas que transcendem as ações e serviços específi- cos de saúde, a exemplo da violência em suas mais diferentes matrizes, o subfinanciamento no âmbito da atenção básica/PSF, limitando o co-financiamento da nesse nível de atenção, aspecto que será analisado a seguir.

\section{A co-responsabilidade do financiamento da aten ção básica/PSF}

H istoricamente, o M inistério da Saúde vem financiando o setor com gasto na doença, onde as ações curativas tomam primazia em detrimento das ações de prevenção e promoção da saúde. Porém, a presença efetiva do PSF em todas as Unidades Federadas e em 5.100 municípios como política nacional para organizar a atenção básica à saúde tem induzido a inversão da lógica vigente do financiamento neste setor.

Uma investida marcante com essa finalidade na busca de novas formas de financiamento ocorreu 
em 1998, quando criou-se o Piso da Atenção Básica (PAB), o qual possibilitou a transferência de recursos calculados em base per capita diretamente aos municípios, acrescendo valores deincentivosfinanceiros vinculados ao número de agentes comunitários de saúde ou de equipes de saúde da família existentes em cada cidade. Diante desses compromissos, nos últimos anos, em consonância com 0 movimento do Governo Federal, também as Secretarias de Estado da Saúde têm, de forma tímida, propiciado incentivos à instalação do PSF, ainda queutilizando diferentes critérios de repasse ${ }^{19}$.

Sabe-se que os resultados no esforço de co-financiamento não podem ser gerados imediatamente, mas já se pode mensurar o seu impacto em alguns estados e municípios. No caso do Governo Federal, percebem-sealguns movimentosqueavançam nesta direção, a exemplo da criação do financiamento da atenção básica - Teto Financeiro do Bloco da Atenção Básica. Constituído pelo somatório das partesfixa evariável do Piso daAtenção Básica, este mecanismo de financiamento foi estabelecido nas diretrizes dos Pactos pela Vida e de Gestão ${ }^{20}$.

Além da definição do teto do financiamento, outros investimentos são direcionados aos municípios com mais de 100 mil habitantes, por meio do Projeto de Expansão e Consolidação do Programa Saúde da Família (PROESF), co-financiado pelo Banco Internacional para a Reconstrução e o Desenvolvimento (BIRD), voltado para a organização e o fortalecimento da atenção básica à saúde no país. Esse investimento éconsiderado no texto de $\mathrm{H}$ eimann e $\mathrm{M}$ endonça ${ }^{21}$ como uma fase de consolidação e expansão planejada do PSF.

As autoras revisam a emergência, o foco e os objetivos do PROESF, com destaque para a importância do PSF enquanto estratégia política na conversão do modelo de atenção à saúde. Afirmam que os recursos destinados pelo BIRD no valor de US $\$ 275$ bilhões de dólares, com igual valor de contraparte do governo brasileiro, ampliam o potencial da implantação do PSF, mesmo em áreas que já tenham oferta de serviços de saúde de outro tipo.

Ressal ta-se, novamente, queapesar dereconhecer os esfor ços na ampliação dos recursos destinados à atenção básica, Estratégia de Saúde da Família, o modelo atual de financiamento, PAB (fixo e variável), não consegue modificar os critérios de distribuição do incentivo federal, principalmente, para os municípios com população acima de 100 mil habitantes, que somam 261, no país²2. Dificulta-se, com isso, a conversão do modelo eo alcance das coberturas almejadas, que correspondem a um mínimo de 70\%, segundo preconiza o PROESF.

N esse sentido, o Gráfico 1 demonstra quehouve um incremento no repasse dos recursos federais do SUS, ao longo dos seis últimos anos, para apoiar os municípios, na responsabilidade de gestor local, na implantação e consolidação da Estratégia de Saúde da Família por meio da expansão e qualificação da atenção básica.

Tal progressão ascendente é visível, tanto nas transferências do PAB fixo, hoje na ordem de R\$

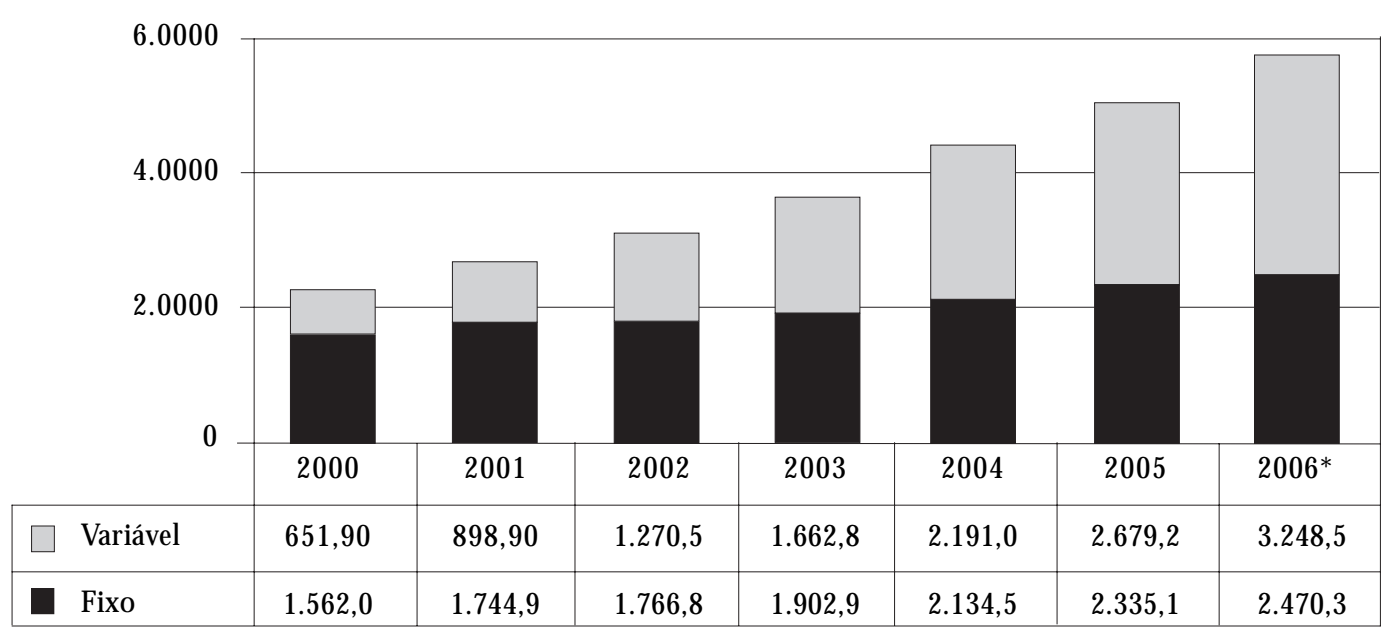

Gráfico 1. Evolução dos recursos financeiros da atenção básica. Brasil, 2000-2006.

(*): Orçamento.

Fonte: Fundo Nacional deSaúde. 
2,4 bilhões, quanto no variável, destinado ao PSF, na ordem de $R \$ 3,2$ bilhões, ainda que muito distante dos valores aplicados na média e alta complexidade, que foram de $R \$ 17,2$ bilhões, segundo dados do Fundo $\mathrm{N}$ acional de Saúde para julho de 2006. Isto não significa que os recursos para uma política universal eintegral não continuem insuficientes com o orçamento recorde de $R \$ 40$ bilhões.

0 que é interessante frisar é que a maior parte dos municípios utiliza, para o custeio das ações e serviços ofertados pelas unidades básicas de saúde da família, todo o recurso do PAB (fixo evariável). Isto porque os recursos transferidos através do incentivo federal não são suficientes para manter as equipes do PSF funcionando. Logo, diante dos pequenos valores repassados para o PSF, os municípios têm destinado recursos próprios adicionais. Porém, essa fonte de recursos tem se apresentado problemática porque a crise das finanças municipais cria barreiras ao gasto com saúde, em geral, e à atenção básica/PSF, em particular.

Assim, em um país em que os municípios têm alto nível de desigualdade de renda e de recursos próprios, pode-se dizer que os incentivos federais cumprem um papel importantena garantia da equidade do gasto do PSF. Não obstante, não se pode afirmar que haja compatibilidade entre os objetivos propostos pela Estratégia de Saúde da Família e a forma de financiá-la, o que requer problematizar alguns pontos dessa questão. Por exemplo, cabenos questionar que medidas as três instâncias gestoras do SUS adotaram para priorizar na peça orçamentária e financeira, nos seus governos e nos subsequentes, a atenção básica/PSF? As medidas adotadas recentemente são suficientes paraa continuidade da re-organização do modelo de atenção à saúde preconizado pelo SUS? Tais medidas incluem a criação do 130 salário-repasse para os Agentes Comunitários de Saúde, alteração da tabela de incentivo de PSF para os municípios com mais de 100.000 habitantes da Amazônia Legal (com IDH menor ou igual a 0,7$)$, municípios onde as equipes atuam em áreas de assentamentos rurais e de quiIombolas e o aumento do PAB, com seu valor mínimo saindo de $R \$ 10,00$ para $R \$ 13,00$.

Encontrar respostas para essas e outras indagações no tocanteà questão do financiamento da atenção básica/PSF nos impõem novos desafios, principalmente com relação à prática co-operativa construída entre os gestores do SUS. Tudo isto lembra a necessidade de definir mecanismos de financiamento que contribuam para a redução das desigualdades regionais e para uma melhor proporcionalidade entreos trêsníveis deatenção do SUS, com primazia para a expansão dos recursos alocados ao $\mathrm{PSF}^{23}$.
N este caso, as esferas de governo, federal e estadual e municipal, devem estabelecer pactos de coresponsabilidade no financiamento das ações e os serviços de saúde, provendo o direito à saúde, de forma responsável e solidária. Com isto, a Estratégia de Saúde da Família - eixo prioritário de investimento da atenção básica - devecontar com financiamento específico, proveniente da responsabilidade constitucional, também, do governo estadual.

A política de capacitação, formação e educação permanente para pessoal do PSF

Para que o PSF não ficasse confinado no "SUS para o pobres" ${ }^{24}$ e na "atenção primitiva de saú$\mathrm{de}^{\prime 25}$, nem sua expansão focalizada apenas nas áreas rurais e periferias das cidades, a Coordenação de Saúde da Comunidade (COSAC) da Secretaria de Assistência à Saúde(SAS) do M inistério, atualmente Departamento de Atenção Básica (DAB), da Se cretaria de Atenção à Saúde, no final do ano de1996, ensejou a implantação de pólos de capacitação, formação e educação permanente para pessoal do Saúde da Família. M ediante edital no 04, de 06/12/96, foram convocadas as instituições de ensino e serviço a apresentarem projetos que possibilitassem uma ação renovada no espaço de articulação ensino-serviço, com vista à capacitação e formação de profissionais capazes de construir o PSF nas diferentes regiões do país.

Essestinham como tarefacentral articular eabrir o diálogo social em torno de uma agen da concreta, capaz de ampliar o debate da mudança na formação dos profissionais da saúde, que, há décadas vem se dando, e para a qual, no dizer de Paim ${ }^{26}, 0$ otimismo não basta. Dessa forma, a SAS/M S traz para a mesa nacional a re-discussão dessa problemática e a possibilidade de encontrar caminhos para seu enfrentamento.

Foram apresentados 21 projetos que, ao serem analisados, resultaram em aprovação de dez como pólos, seis como projetos de capacitação e cinco como projetos de cursos instalados. Os projetos que não se caracterizaram como pólos e sim como centros de capacitação e cursos introdutórios desempenharam um importante papel na preparação de RH para o PSF, cuja agenda foi construída a curto, médio e longo prazo, considerando os seguintes compromissos: (1) oferta de cursos de atualização para médicos, enfermeiros, auxiliares de enfermagem e agentes comunitários de saúde, ou outros profissionais que integrem a equipe de saúde da família, devendo ser incluídas obrigatoriamenteas categorias de médico e de enfermeiro; (2) acompanhamento e avaliação do trabalho das 
equipes nas Unidades Básicas de Saúde da Família; (3) implantação de programas de educação permanente destinados aos profissionais da equipe de saúde da família existente em um município ou conjunto de municípios, utilizando como instrumentos cursos curtos presenciais, educação à distância ou atualização, valendo-se das atividades de interconsultas a especialistas, recursos audiovisuais e de telemática e outros; e (4) implantação de cursos de especialização ou outras formas de cursos de pós-graduação lato sensu para médicos, enfermeiros e outros profissionais que atuam no Programa Saúde da Família, iniciativa esta destinada a introduzir inovações curriculares nos cursos de graduação.

A complexidade desses compromissos sinalizava para a necessidade de ampliação da rede de instituições parceiras envolvidas com os pólos. Para tanto, em 1998, a Secretária de Assistência à Saúde (SAS/M S) lança um novo edital, estimulando que cada Unidade da Federação tomasse para si a responsabilidade defortalecer a rede pólo no estado e no conjunto de suas regiões/municípios.

Os sujeitos dirigentes do PSF à época estavam atentos ao que afirmava Paim²7: " [...] os pólos não merecem limitar-se a quistos de universidades, enquanto vitrines de mudanças virtuais". Diante dessealerta, verificou-sea necessidadedeacompanhar os resultados daqueles pólos. E, para tanto, foram realizadas algumas pesquisas, a exemplo do Perfil dos M édicos e Enfermeiros do PSF ${ }^{28}$.

Os resultados dessas pesquisas foram utilizados para subsidiar as negociações e definições de linhas estratégicas de ação no que diz respeito à formação, capacitação e educação permanentejunto ao M inistério da Educação e outras instâncias governamentais.

Em 2003, estes pólos foram substituídos por uma nova proposta que incluía todos os trabal hadores do SUS, os usuários da rede de serviços, a rede formadora e os estudantes, denominada Pólos de Educação Permanente para os trabalhadores do SUS. A implantação dessa nova proposta não foi precedida de qual quer avaliação dos resultados da experiência anterior que permitisse apreender os seus limites e as possibilidades de potencialização daquilo que havia de agregador em ambas as propostas. Uma nova conjuntura institucional determinou a suspensão das experiências dos "pólos", tanto do PSF quanto da ampliação dos mesmos para o restante do SUS.

0 que se aprende com fatos dessa natureza é que sujeitos públicos, ocupando cargos governamentais, podem perder a racionalidade na formulação das políticas públicas, tratando-as como uma ação conjuntural, em vez de uma ação estruturante de um Estado- nação. N essa condição, o tempo e o cuidado com os sujeitos envolvidos nas políticas em curso são elementos essenciais para a consolidação das estratégias que implicam mudanças concretas.

De acordo com as considerações de Paim ${ }^{27}$ : "Uma universidade e uma faculdade de medicina não são uma planta industrial capaz de alterar rápida eimpunementesuaslinhas demontagem [...]". Concordando com o autor, acrescentamos que esses espaços são constituídos por sujeitos estratégicos, comprometidos e protagonistas de um projeto radical da reforma sanitária e da reforma do ensino, e que merecem ser vistos na suas capacidades de produzir mudanças, especialmente se elas são construídas a partir dos saberes coletivos e dos poderes compartilhados. M ovimentos em sentido oposto podem trazer incompreensões, equívocos e frustrações.

Conforme se pode depreender desse fato, a estratégia de capacitação, formação e educação permanente para o pessoal do PSF continua sendo uma questão a ser enfrentada, enquanto uma política de Estado capaz de levar adiante mudanças em curso na formação, sobretudo dos profissionais da medicina. Busca-seque esses profissionais estejam preparados, e comprometidos, para exercer a prática da saúde integral, familiar e comunitária.

Ainda, essa responsabilidade cabe primeiramente ao M inistério da Saúde, que deve cumprir os dispositivos constitucionais conforme 0 artigo 200, inciso III - ordenar a formação de recursos humanos na área da saúde ${ }^{29}$, exercendo o papel de indutor junto às secretarias estaduais e municipais de saúde, e principalmente às instituições de ensino superior. Essas instituições não mediram esforços no sentido de envidar energias, crenças e esperanças na construção de outro modelo deatenção à saúde das famílias brasileiras.

E essa construção não pode prescindir de uma outra questão desafiadora, a de produzir tecnologias gerenciais, aplicadas aos processos de monitoramento e avaliação, colocando no centro da discussão os seguintes aspectos: a qualidade da expansão das equipes do PSF, a utilização do sistema deinformação como ferramenta gerencial, que contemplea complexidade da organização da atenção e apresente indicadores adequados e oportunos para o monitoramento e avaliação dos efeitos do PSF nos municípios brasileiros.

Tamanha tarefa exige a institucionalização de processos estruturados e sistemáticos de avaliação dessa estratégia, nas suas mais variadas dimensões como, por exemplo, atenção à saúde, gestão, im- 
pacto sobreo perfil epidemiológico ea participação social. Esta última dimensão foi relembrada por Teixeira ${ }^{30}$, ao citar Maria Cecília Donnangelo, que nos ensinou em 1979, no tocante à reforma, "se fará com a população ou não se fará". Isso nos faz "aprender a aprender" que as tecnologias aplicadas aos processos de monitoramento e avaliação de vem estar entrelaçadas com o compromisso ético, humanístico e social com asfamílias cuidadas pelas equipes do PSF, nos seus espaços de convivência.

Por fim, novas práticas de saúde devem ser orientadas pela essência do SUS. Há que se fortalecer o diálogo de forma permanente, franca e democrática, com todas as forças sociais, tendo em vista um novo pacto federativo: a consolidação da política prevista na nossa Constituição. Concordamos queÉ preciso vencer as muralhas do liberalismo econômico, dos privilégios de classes, dos corporativismos queà medida queo sistema único de saúdeavança oporão uma resistência cada vez mais encarniçada aberta ou disfarçada ${ }^{31}$. No caso do PSF, tal disfarce é potencializado pela inércia de uma agenda incompleta.

Incompleta porque não conseguiu, ao longo dos doze anos de implantação e implementação, ampliar as condições para a superação efetiva do modelo biomédico hegemônico nas políticas públicas do setor saúde no Brasil.
Colaboradores

M F Sousa foi responsável pela concepção e redação do artigo eEM Hamann cuidou da metodologia e da revisão final do artigo.

\section{Referências}

1. Brasil. M inistério da Saúde. Sistema de Informação da Atenção Básica (SIAB). Brasília: Ministério da Saúde; 2006.

2. Donnangello MCF. Saúde e sociedade. São Paulo: Duas Cidades; 1976.

3. Arouca ASS. 0 dilema preventivista: contribuição para compreensão e crítica da medicina preventiva [tese]. Campinas (SP): Faculdade de Ciências M édicas, Universidade Estadual de Campinas; 1975.

4. García JC. Pensamento social em saúde na América Latina. São Paulo: Cortez; 1989.

5. M endes-Gonçalves RB. M edicina e História: as raízes sociais do trabalho médico [dissertação]. São Paulo (SP): Departamento de M edicina Preventiva, Universidade de São Paulo; 1979.

6. Brasil. Senado Federal. Constituição da República Fe derativa do Brasil. Brasília: Senado Federal; 1988.

7. Brasil. Lei $n^{\circ} 8.080$ de 19 de setembro de 1990. Dispõe sobre as condições para a promoção, proteção e recuperação da saúde, a organização e o funcionamento dos serviços correspondentes e dá outras providências. Diário Oficial da União 1990; 19 set.

8. Brasil. Lei no 8.142 de 28 de dezembro de 1990. Dispõe sobre a participação da comunidade na gestão do Sistema Único de Saúde e sobre as transferências intergovernamentais de recursos financeiros na área da saúde e dá outras providências. Diário Oficial da União 1990; 28 dez.

9. Starfield B. Atenção primária: equilíbrio entre necessidades de saúde, serviços e tecnologia. Brasília: UNESCO/M inistério da Saúde; 2002.

10. Mendes EV. Uma agenda para a Saúde. São Paulo: Hucitec; 1996. 
11. Gentile M. Promoção da Saúde. Brasil. Revista brasileira em promoção da saúde 1999; 1(1):20-32.

12. Brasil. Portaria no 3.925 de 13 de Novembro de 1998, que aprova o Manual para Organização da Atenção Básica no Sistema Ú nico de Saúde. Diário Oficial da União 1998; 13 nov.

13. Brasil. Portaria no 648 de 28 de março de 2006, que aprova a Política Nacional de Atenção Básica, estabelecendo a revisão de diretrizes e normas para a organização da Atenção Básica para o Programa Saúde da Família (PSF) e o Programa Agentes Comunitários de Saúde (PACS). Diário Oficial da União 2006; 29 mar.

14. Campos FE, Cherchiglia ML, Aguiar RAT. Reflexões sobre Saúde da Família no Brasil: desafios e oportunidades. Revista brasileira de saúde da família 2002; 2(5):71-79.

15. Teixeira CF. A mudança do modelo de atenção à saúde no SUS: desatando nós, criando laços. Saúde em debate 2003; 27(65):257-277.

16. Viana ALA, Dal Poz MR. A reforma do Sistema de Saúde no Brasil e o Programa de Saúde da Família. Physis 1998; 8(2):11-48.

17. Sousa M F, Fernandes AS, Araujo CL, Fernandes MC. Gestão da atenção básica: redefinindo contexto e possibilidades. Divulgação em saúde para debate 2000; 21:7-14.

18. Teixeira CF. A mudança do modelo de atenção à saúde no SUS: desatando nós, criando laços. Saúde em debate 2003, 27(65):257-277.

19. Sousa MF, organizadora. Os sinais vermelhos do PSF. São Paulo: Hucitec; 2002.

20. Brasil. Portaria no 699 de 30 de março de 2006, que regulamenta as Diretrizes Operacionais dos Pactos pela Vida e de Gestão. Diário Oficial da União 2006; 31 mar.
21. Heimann LS, M endonça MH . A trajetória da Atenção Básica em Saúde e do Programa Saúde da Família no SUS: uma busca de identidade. In: Lima NT, Gerschman S, Edler FC, Suárez JM, organizadores. Saúde e democracia: história e perspectivas do SUS. Rio de Janeiro: Fiocruz; 2005. p. 481-502.

22. Instituto Brasileiro de Geografia e Estatística. Censo Geográfico e Estatístico. Brasil; 2000. [acessado 2006 dez]. Disponível em: http://www.ibge.gov.br/censo/

23. Sobrinho EJMA, Sousa MF. O PSF nos grandes municípios: São Paulo, um investimento à parte! In: Sousa $M F$, organizadora. Os sinais vermelhos do PSF. São Paulo: Hucitec; 2002. p.47-68.

24. Paim JS. Formação médica e políticas de saúde. In: Anais do XXXIII Congresso Brasileiro de Educação M édica. Porto Alegre; 1995. p. 247-261.

25. Testa M. Atenção Primária (ou primitiva?). In: Testa M, organizador. Pensar em saúde. Porto Alegre: Artes M édicas; 1992.

26. Paim JS. Educación médica en la década de 1990: el optimismo no basta. Educ M ed Salud 1991; 25(1):48-57.

27. Paim JS. Saúde, política e reforma sanitária. Salvador: CEPES/ISC; 2002.

28. Brasil. M inistério da Saúde. Perfil dos médicos e enfermeiros do PSF. Brasília: Ministério da Saúde; 2000.

29. Brasil. Senado Federal. Constituição da República Federativa do Brasil. Brasília: Senado Federal; 1988. p. 134.

30. Teixeira CF. A mudança do modelo de atenção à saúde no SUS: desatando nós, criando laços. Saúde em debate 2003; 27(65):274.

31. Sobrinho EJMA, Sousa MF. A tarefa: apagar os sinais vermelhos do PSF. In: Sousa M F, organizadora. Os sinais vermelhos do PSF. São Paulo: Hucitec; 2002. p.155-170. 